\title{
SIRT1 activity is decreased in lesional psoriatic skin
}

\author{
Matteo Becatti ${ }^{1}$ Victoria Barygina ${ }^{1}$ Giacomo Emmi ${ }^{2} \cdot$ Elena Silvestri $^{2}$. \\ Niccolò Taddei $^{1} \cdot$ Torello Lotti $^{3} \cdot$ Claudia Fiorillo $^{1}$
}

Received: 6 May 2016/Accepted: 1 June 2016

(C) SIMI 2016

\section{Dear Editor,}

Psoriasis is a chronic immune-mediated hyperproliferative inflammatory skin disease of unknown etiology characterized by the appearance of sore patches of thick, red skin with silvery scales. It affects about up to $3 \%$ of the world population and it is equally prevalent in both genders, although results from a recent study have shown that on average, men have more severe forms of the disease.

Psoriasis treatment often requires a varied team of clinicians with a range of expertise due to the fact that high prevalence, chronicity, disfiguration, disability, and associated comorbidity characterize the disease. The deep understanding of the role of immune function in psoriasis permits the management of this complex disease, which dramatically affects patients far beyond the skin.

Hence, psoriasis is associated with impairment in health related quality of life, and, recently, it has emerged as a systemic disease, which affects not only cutaneous and articular sites, but it can also result in metabolic impairments and adverse cardiovascular outcomes [1]. Cytokines and growth factors released by activated $\mathrm{T}$ cells have been shown to display a prominent role in keratinocyte hyperproliferation; however, we focused our attention on dermal

M. Becatti and V. Barygina are equally contributed.

Matteo Becatti

matteo.becatti@unifi.it

1 Department of Experimental and Clinical Biomedical Sciences "Mario Serio", University of Florence, Largo Brambilla 3, 50134 Florence, Italy

2 Department of Clinical and Experimental Medicine, University of Florence, Florence, Italy

3 Dermatology and Venereology Division, Guglielmo Marconi University, Rome, Italy fibroblasts, which are also directly involved in the developing psoriatic lesions accelerating keratinocyte proliferation [1].

Emerging research suggests that reactive oxygen species (ROS) play a central role in the pathogenesis of psoriasis. Indeed, a number of studies reveal an altered blood redox status in psoriatic patients as shown by significantly increased levels of oxidative stress markers and decreased activity of the main antioxidant enzymes [2]. SIRT1, a mammalian ortholog of Silent information regulator 2 (Sir2) family, is significantly upregulated in response to oxidative stress [3]. Sirtuins are a family of $\mathrm{NAD}^{+}$-dependent protein deacetylases that are evolutionally conserved from yeast to human. SIRT1, the most extensively studied member of the sirtuin family, targets a number of substrates-histones, transcription factors, DNA repair proteins, autophagy factors, and others-to modulate metabolism, stress responses, and many other cellular processes [3]. We have previously demonstrated that SIRT1 activation regulates mitogen-activated protein kinases (MAPK) pathway and down-regulates pro-apoptotic molecules, decreasing oxidative stress and reducing apoptotic cell death in keratinocytes from vitiligo skin.

In the literature, there is neither evidence for SIRT1 expression and activity in lesional psoriatic fibroblasts, nor for its possible involvement in oxidative stress-related pathways. Here, for the first time, we investigated SIRT1 expression and activity in a fibroblast primary culture from lesional psoriatic skin, and we hypothesize its involvement in oxidative-mediated alterations.

The study was approved by the Local Ethics Committee, and carried out according to the Helsinki Declaration. Analyses were carried out in eight patients (four females and four males) affected by moderate psoriasis $(\mathrm{PASI}=12.5 \pm 0.5)$ with a mean age of $40.1 \pm 8.7$ years and with a mean duration of disease of 15.8 years (from 8 
Table 1 Demographic and clinical data of patients involved in the study

\begin{tabular}{lllll}
\hline Patients & Age & BMI & Duration of disease (years) & PASI \\
\hline M1 & 33 & 25 & 8 & 13 \\
M2 & 41 & 24 & 12 & 13 \\
M3 & 29 & 25 & 10 & 12 \\
M4 & 23 & 21 & 12 \\
F1 & 52 & 21 & 25 & 13 \\
F2 & 45 & 23 & 20 & 13 \\
F3 & 38 & 26 & 18 & 12 \\
F4 & 32 & 24 & 12 & 12 \\
Mean \pm SD & $40.1 \pm 8.7$ & $23.9 \pm 1.6$ & $15.8 \pm 6.1$ & $12.5 \pm 0.5$ \\
\hline
\end{tabular}

a

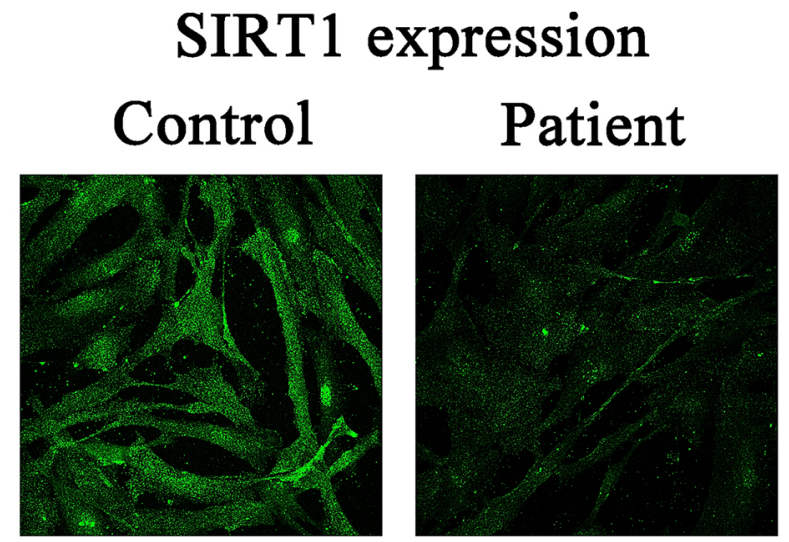

b
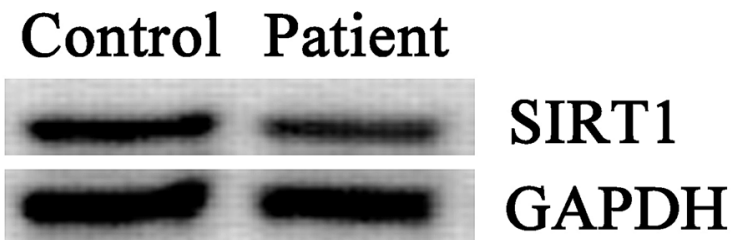

C

\section{SIRT1 activity}

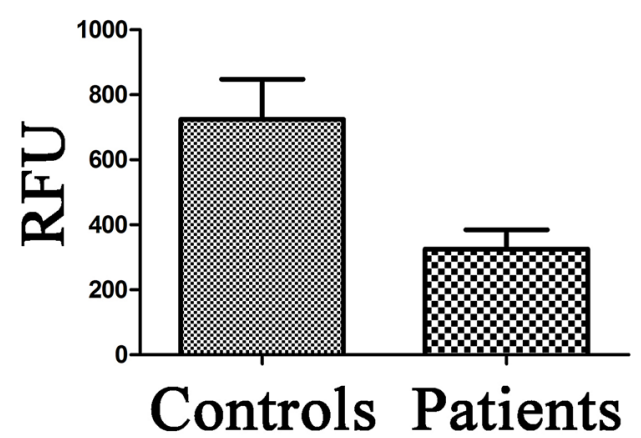

Fig. 1 Confocal microscope (a), western blot (b) analyses of SIRT1 expression and SIRT1 activity (c) in a fibroblast primary culture from lesional psoriatic skin and healthy controls to 25 years). The demographic and clinical information for each patient are summarized in Table 1 . Ten non-stressed healthy controls (five females and five males), matched for age and body mass index, were also enrolled in the study. No subjects involved in the study followed any systemic therapy before the study, or had a history of any disease, e.g., diabetes mellitus and atherosclerosis, which might affect the redox status.

SIRT1 expression was assessed by confocal microscopy analysis (a) and quantified by western blot (b). As shown in Fig. 1a, SIRT1 expression is markedly reduced in fibroblasts from psoriatic patients compared to healthy subject; in particular, western blot analysis confirms the reduction of SIRT1 expression in psoriatic fibroblasts compared to controls ( $-43 \pm 5 \%$ vs Controls).

SIRT1 activity was assessed using SIRT1 Direct Fluorescent Screening Assay Kit (Cayman, Ann Arbor, MI, USA) as previously described [4]. Compared with fibroblasts from healthy subjects, psoriatic cells exhibit a marked decrease $(325.8 \pm 58.42$ vs $724.3 \pm 123.9, p<0.0001)$ in SIRT1 activity.

This is the first evidence for a reduced SIRT1 expression and activity in lesional psoriatic fibroblasts, suggesting the involvement of SIRT1 in the pathogenesis of psoriasis. Many aspects remain to be elucidated; however, therapeutic strategies based on SIRT1 activators might also be taken into consideration to control the metabolic and cardiovascular derangement of the disease [5].

Compliance with ethical standards

Conflict of interest The authors declare that they have no conflict of interest.

Statement of human and animal rights The study protocol was approved by local Ethical Committee.

Informed consent Informed consent was obtained from all enrolled subjects. 


\section{References}

1. Boehncke WH, Schön MP (2015) Psoriasis. Lancet 386:983-994

2. Zhou Q, Mrowietz U, Rostami-Yazdi M (2009) Oxidative stress in the pathogenesis of psoriasis. Free Radic Biol Med 47:891-905

3. Alcendor RR, Gao S, Zhai P, Zablocki D, Holle E, Yu X, Tian B, Wagner T, Vatner SF, Sadoshima J (2007) SIRT1 regulates aging and resistance to oxidative stress in the heart. Circ Res 100:1512-1521
4. Becatti M, Fiorillo C, Barygina V, Cecchi C, Lotti T, Prignano F, Silvestro A, Nassi P, Taddei N (2014) SIRT1 regulates MAPK pathways in vitiligo skin: insight into the molecular pathways of cell survival. J Cell Mol Med 18:514-529

5. Esser N, Paquot N, Scheen AJ (2015) Anti-inflammatory agents to treat or prevent type 2 diabetes, metabolic syndrome and cardiovascular disease. Expert Opin Investig Drugs 24:283-307 